\title{
DEVELOPING A GENERALISED ASSESSMENT FRAMEWORK FOR RAILWAY INTERVENTIONS
}

\author{
JOHN ARMSTRONG ${ }^{1}$, GEORGIOS REMPELOS ${ }^{1}$, JINGFU WEI $^{2}$, JOHN PRESTON ${ }^{1}$, \\ SIMON BLAINEY ${ }^{1}$, JOHN EASTON ${ }^{2} \&$ CLIVE ROBERTS $^{2}$ \\ ${ }^{1}$ Transportation Research Group, University of Southampton, UK \\ ${ }^{2}$ Birmingham Centre for Railway Research and Engineering, University of Birmingham, UK
}

\begin{abstract}
Work undertaken on the socio-economic assessment of the installation of under-sleeper pads (USPs) on a busy commuter railway route in Britain, as part of the Track to the Future (T2F) project, was presented at COMPRAIL 2018. This was followed by an expanded, comparative analysis of the same route, comparing the noise-reduction and other costs and benefits of USPs, rail dampers and noise barriers, published in the International Journal of Transport Development and Integration. Such analyses provide useful information and decision-making assistance to infrastructure managers, funders and other stakeholders, but typically require the repeated development of bespoke models of study locations, interventions and effects. The "one-off" nature of these models increases the time and costs required for such studies, and makes it more difficult to compare similar schemes in different locations, or different types of intervention in a single location. There is, therefore, a need for an improved, more generalised and standardised, transferable, replicable and comparison-enabling approach to the socioeconomic assessment of such interventions. Ongoing work on the T2F project entails the development and use of standardised templates to describe the input costs and expected impacts of different infrastructure, rolling stock or operational interventions. These form the inputs to, and part of the development for, T2F of an improved, generalised, ontology-based assessment framework, which is being developed using the West Coastway railway line between Portsmouth and Brighton on England's south coast as an initial case study. This paper describes the context and rationale for the development of the assessment framework, its components and the methodology being adopted.
\end{abstract}

Keywords: railways, maintenance, renewals, enhancements, interventions, environmental impact, economic assessment, assessment template, ontologies.

\section{INTRODUCTION}

Recent decades have seen significant growth in railway traffic in Britain, as elsewhere in Europe and beyond [1], [2]. Recent changes in annual train kilometres in Britain are shown in Fig. 1, based on data published by the Office of Rail and Road (ORR) [3], [4], with growth in passenger train kilometres partly offset by recent declines in freight train kilometres. Such traffic growth is welcome for the railway industry, following a long period of decline in the mid-20th century, but can also present it with capacity challenges. Similarly, while modal shift to rail (especially from road and air travel) is generally desirable on environmental grounds, it does have its own environmental impacts, notably including the effects of noise and vibration on non-users living or working close to railway lines, which increase with traffic growth and may need to be reduced at source and/or attenuated.

In addition to such enhancements, the growth in traffic levels results in an increased need for infrastructure maintenance and renewals, and less opportunity to carry out any of these necessary works. There is therefore also a need for improved planning and implementation of maintenance and renewals (M\&R) activities [5], and, more generally, for interventions to increase infrastructure working life. In order to achieve the desired outcomes in the most cost-effective manner, there is also a need for methods and tools to assess the relative costs and benefits of alternative approaches to such interventions. Previous work undertaken in the 


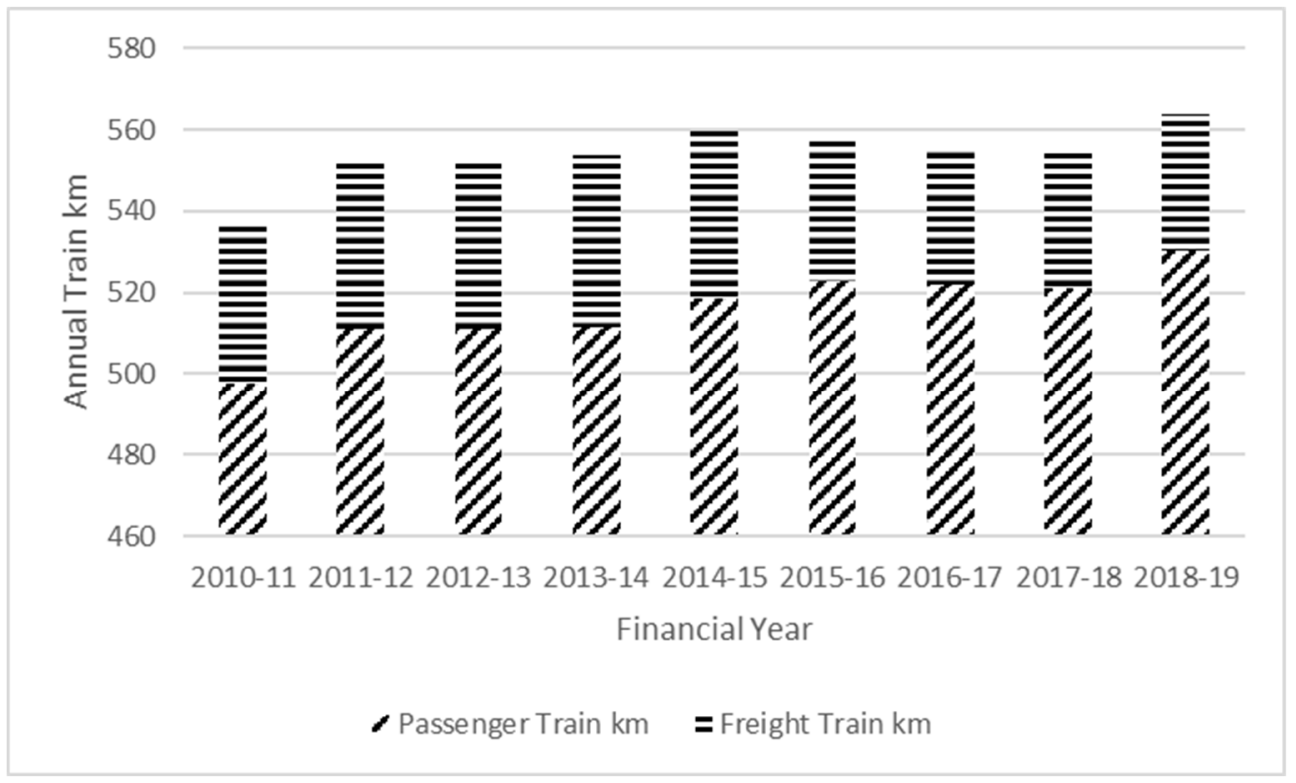

Figure 1: Annual train kilometres in Britain, 2010-2011 to 2018-2019 [3], [4].

Track21 [6] and Track to the Future (T2F) [7] research programmes, whose aims are to improve the "engineering, economic and environmental performance of railway track" in Britain, developed bespoke cost-benefit analysis (CBA) tools for specific infrastructure interventions, as described in Ortega et al. [8] and Armstrong et al. [9].

While the outputs of these exercises provided useful information, and Armstrong et al. [9] included comparative analysis of alternative interventions, rather than assessing a single "do something" option against a "do minimum" base, the approaches used were still somewhat location- and situation-specific. It thus became apparent that, rather than conducting repeated, one-off assessments of possible interventions, there are significant potential benefits to be gained from the development of a standardised, flexible assessment framework and methodology, capable of assessing different types of intervention on different parts and extents of the national (or another) railway system. This paper sets out the approach being adopted to deliver these benefits.

Following this introduction, the background to and context of the work are first set out, including the rationale for the proposed new approach. The two main elements of the updated approach are then described. This is followed by a description of the case study being used for the initial implementation of the approach. Ongoing and further work are then described, followed by some conclusions and a list of references.

\section{BACKGROUND AND CONTEXT}

As indicated in the Introduction, considerable work has been done in the Track21 and T2F research programmes to develop methods and tools to model the whole-life costs and benefits of a range of infrastructure interventions. Much of the focus was on the reduction of environmental impacts (chiefly noise and vibration) on non-rail users (for example through the installation of under-sleeper pads (USPs), rail dampers or noise barriers), but the wider impacts on track quality, whole-life costs and $M \& R$ requirements were also considered, as 
were the effects on ride quality for rail users. There is considerable debate surrounding the economic valuations of noise and vibration reductions, and approximations are required to estimate the numbers of people affected by interventions, and the extent to which they benefit from or are disadvantaged by the resulting changes. These and other uncertainties (including valuations of ride quality changes) have been addressed by using Monte Carlo simulations and sensitivity analyses to identify a likely range of potential impacts.

Again, this approach has been applied to the assessment of a range of interventions in different locations, but, even for the comparative studies of alternative interventions in a single location, repeated and time-consuming, one-off analyses were required to obtain the required outputs, reducing the potential replicability of the approach for different interventions and locations, and the direct comparability of the results obtained. One of the wider objectives of the T2F research programme, therefore, is the development of a standardised framework for the assessment of the economic (and, ultimately, carbon) impacts of different infrastructure (and other, e.g. rolling stock) interventions in different locations and operating circumstances. Work to date to meet this wider objective has focussed on the use of standardised input "templates" for different interventions, and on moving towards an ontological approach to the handling and processing of the intervention data; these two aspects of the work are considered in the following sections of this paper.

\section{STANDARDISED INPUT TEMPLATES}

In a large research programme like T2F (and often more generally, in the assessment of large infrastructure and transport interventions and investments), the analysis of economic and carbon impacts tends to be undertaken by specialists in those fields, relying upon expert advice and input on the nature, costs and impacts of individual interventions. It is helpful to both parties if the data requirements for input to the economic and carbon assessments are clearly stated and specified, and the resulting inputs are of a standardised (or as much as is possible in the context of different interventions) layout and format.

A standardised input template was produced in an iterative process, by means of development of initial proposals, discussion with and feedback from colleagues, and subsequent refinement; the current version of the template is included as an Appendix, and its main features are summarised below.

Following a description of the type of intervention under consideration and the name of the investigator, the template consists of six main, generally qualitative, questions (questions 3-6 have associated sub-questions, seeking associated quantitative information). Additional comments are also elicited. The six main questions and associated sub-questions are:

1. Why will this intervention improve rail track systems?

2. In assessing the engineering impacts of this intervention what are the key input and output variables?

3. What are the main one-off (capital expenditure) and recurrent (operating expenditure) financial costs of the intervention?

Sub-question: What is your judgement of the magnitude of these costs and can they be quantified? If so, how?

4. What are the main operator benefits? Operator refers to both infrastructure (Network Rail and its suppliers) and train services (train operating companies and their suppliers).

Sub-question: What is your judgement of the magnitude of these benefits and can they be quantified? If so, how? 
5. What are the main user benefits? User refers to customers of passenger and freight train services.

Sub-question: What is your judgement of the magnitude of these benefits and can they be quantified? If so, how?

6. What are the main non-user benefits? Non-user refers to users if rival transport systems (air, road), residents or the wider community.

Sub-question: What is your judgement of the magnitude of these benefits and can they be quantified? If so, how?

The template thus seeks a set of standardised qualitative inputs to the assessment of a proposed intervention, supported where possible by the associated quantitative inputs needed for detailed economic and/or carbon analysis. It is not generally anticipated that the subject matter expert for a given intervention will be able to directly provide all the data inputs needed for detailed analysis, but rather that (s)he will be able to "point the analysts towards" the sources of the required data.

\section{AN ONTOLOGICAL APPROACH}

\subsection{The need for a new approach}

Having developed a standardised template (informed by logic mapping, as in The Magenta Book [10]) for intervention-related inputs, outputs and outcomes/impacts, attention turned to the best means of standardising the analysis process, and developing a transferable, flexible framework to generalise the modelling processes. These processes should consider and include the whole life socio-economic (all relevant costs and benefits including carbon) and financial (all relevant cash flows, including Schedule 4 and 8 compensation payments for, respectively, planned and unplanned disruptions) impacts of track/railway system interventions, i.e. changes to infrastructure and/or the traffic using it.

It was envisaged that the result should take the form of a "whole-life cost dashboard", ideally interfacing with existing tools such as the Rail Safety and Standards Board's (RSSB's) whole life carbon model, and potentially contributing to a "digital twin" of the railway system. This would provide improved understanding, and enable easier and more effective management and handling, of industry datasets, including, for example, the identification, tagging and handling of missing data. Such a generalised lifecycle cost model would hold and process data from different sources and provenances, and, where necessary, of variable quality and criticality (i.e. the precision and accuracy of some datasets are more important than those of others).

In order to meet these needs, it was decided that an ontological approach should be adopted, as described and advocated in Morris and Easton [11] as a suitable means of integrating and processing disparate sources of railway data to generate useful information and knowledge. Ontologies (in the field of computer science, rather than philosophy) are used to study the existence of entities within a specific domain. Perhaps most easily described as the "knowledge model" component of what has previously been referred to as an Expert System, ontologies display some similarities with well-designed databases in terms of their ability to manage facts relating to the same entity, but with added functionality for the capture of semantics (i.e. data meaning, source, quality, shortcomings, etc.). Semantics and rules describing the relationships between concepts (e.g. different measures of temperature and the conversions linking them) are stored in models with the corresponding data, and 
transformations and translations then be undertaken within models, enabling updates or translations to be made once, within the scope of the model, rather than in multiple end-user software packages. This is of particular potential value in the context of Britain's railway industry, which uses and relies upon multiple poorly- or non-integrated legacy data systems; each system can request the data in the form it needs, and the model can be relied upon to provide either the raw data as stored (if it is already in the correct form), or to apply the appropriate translation to the data it does hold. From an implementational perspective, ontologies are typically represented using data "triples", a node-link-node structure comprising Observation-Value-Data, or Subject-Predicate-Object (or, in object-oriented programming terms, entity/instance-property-value): for example, "South West Main LineElectrification Type-Third Rail DC". Graph-based databases known as triplestores are then used to hold the complete set of facts of interest, and to manipulate them efficiently.

In the context of the T2F and successor projects, an ontological approach will enable the combination of multiple and varied data sources into a more useful whole; this approach is particularly useful in broad analytical contexts, as in the case under consideration, i.e. the linking of infrastructure and/or rolling stock data with economic and/or carbon assessment.

\subsection{Initial ontology development}

As a first step in the movement towards the development and application of an ontological approach to the problem domain, an intervention modelling framework was developed, with six "top level" areas of primary interest:

- Impact categories (general);

- Inputs;

- Processes;

- Outputs;

- Outcomes;

- Impacts.

These areas were disaggregated and sub-categorised, as set out below, including their subdivision into "Real World" and "Scientific/Experimental" sub-categories where appropriate.

- Impact Categories (general)

$\circ \quad$ Benefits - quantitative (e.g. track life increase, noise reduction) and qualitative

○ Disbenefits - quantitative (e.g. whole-life carbon footprint increase, maintenance cost increase) and qualitative

$\circ \quad$ Additional comments (qualitative)

- Inputs

"Real World"
- Time
- Roney
- Railway Industry Data
- Timetables (base cases)
- Unit Cost data - e.g. USPs, fibre-reinforced ballast, M\&R activities
- Primary process-specific data related to the manufacturing of different
$\quad$ track components 
- Secondary manufacturing and process-specific data

- Detailed qualitative and quantitative data on the installation, maintenance, inspection and renewal procedures adopted by Network Rail (the infrastructure manager of Britain's railways)

- Detailed information related to the end-of-life pathways of different track components (qualitative and quantitative), including locations of landfill sites, energy recuperation facilities, etc. from which detailed data may be obtained

- $\quad$ Other Network Rail financial data: track access charges, schedule 4 and 8 payments, impact on Regulatory Asset Base etc.

- Operational data - train movements (e.g. equivalent million gross tonnes per annum (EMGTPA))

- Operator data: traffic levels (passenger kilometres, tonne kilometres, revenue)

- Scientific/Experimental (exogenous to the intervention)

- Demographics

- Land use

- Railway topography (e.g. embankment vs. cutting)

- Asset condition and characteristics (i.e. what type of asset is it?)

- Ambient air- and ground-borne noise levels

- Carbon emissions by mode

- Aspects of other modes - e.g. road congestion

- Values of time, comfort, etc. - from e.g. WebTAG [12], although some values may be experimental and thus endogenous to an intervention

- Scientific/Experimental (endogenous to the intervention)

- Computational experiments

- Laboratory tests

- Field trials

- Continuous measurements

- $\quad$ Processes

○ "Real World"

- Use

- Degradation

- Maintenance and Renewals

- Enhancements

- Scientific/Experimental

- Operational models

- Economic models

- Environmental models (primarily lifecycle analysis, based on ISO14000 series of environmental standards - in all cases, capturing and representing the results of detailed analyses of micro-level behaviour in macro-scale models is a challenge)

- GIS modelling - data processing and integration

- Outputs

○ "Real World" 
- Engineering interventions - e.g. volume of USP installations, random fibre reinforcement etc.

- Scientific/Experimental

- Calculated costs - one-off (capital) and recurring (operating)

- Calculated operator benefits (to Network Rail, operators and other industry suppliers)

- Calculated user benefits (to passengers and freight customers)

- Calculated non-user benefits (to local residents/workers, users of other modes, wider community)

- Results of financial cost-benefit analysis

- Results of social cost-benefit analysis

- Results of carbon cost-benefit analysis, possibly including other, related impact factors

- Outcomes

○ "Real World"

- Longer track life

- Reduced maintenance

- Reduced noise

- Better track geometry

- Scientific/Experimental

- Extended and improved knowledge and understanding

- Impacts

○ "Real World"

- Reduced maintenance costs, offsetting increased capital costs, and improved benefit:cost ratios (as indicated by the scientific/experimental outputs)

- Improved user and non-user satisfaction

- Scientific/Experimental

- Application of research findings

The modelling framework and the standardised input template were then compared to identify and remedy any "gaps" between the two, and used to identify the available and potential data sources to provide the necessary inputs to the modelling process.

The impact categories, inputs, processes, outputs, outcomes and impacts are being structured in an ontology, building upon the existing Railway Core Ontologies (RaCoOn) [13] and ensuring compatibility with standard terminology used in life cycle costing standards and in WebTAG [12]. Created as a domain-specific representation of the railways, RaCoOn was originally developed to provide a model of the railway track and signalling, which could be used to automatically infer the set of routes available through a junction based on the status of the physical assets present. The model soon expanded to serve a broader range of railway use cases, with a "core" of generic railway concepts that are supported as needed by models for specific subdomains, such as infrastructure, signalling, and rolling stock [13]. The main strength of an ontology-based approach to modelling lies in its use of concepts to structure data. By providing a concept of a "track" that is then further refined by the data used to describe it, for example, $\mathrm{RaCoOn}$ is able to accommodate a wide range of viewpoints around the correct terminology and granularity of representation of that asset. This makes the ontology an ideal basis for the integration of data across different IT systems, with the fact-based, semantic representation of the data used by RaCoOn able to be translated 
to and from a wide range of other contexts without the introduction of ambiguity. In the work described in this paper, RaCoOn is being used to integrate GIS, ambient noise, and wheelrail interaction data from a variety of systems, to enable the workflow shown in Fig. 2.

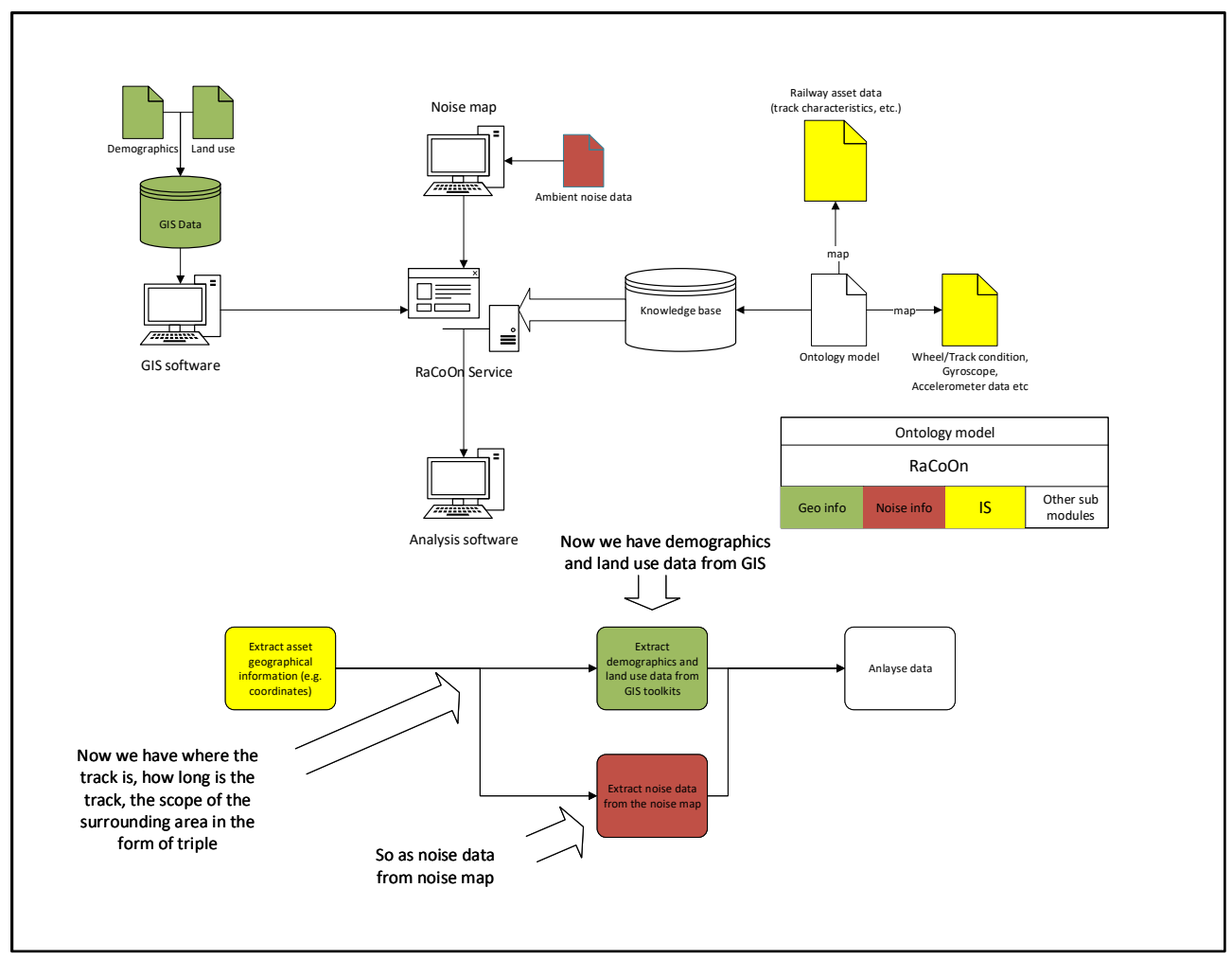

Figure 2: Workflow diagram.

The flow starts from the capture of railway asset data, wheel/track condition, etc. from various sources. A plugin is used to export relevant geographical data shapefiles, which converts geographical data to triples with reference to concepts defined in $\mathrm{RaCoOn}$ and stores them in the triplestore. Mapped data can then be handled by RaCoOn services.

RaCoOn services is a set of tools that helps to store converted data and subsequently retrieve it from storage. Its main purpose is to help users to retrieve data from storage and export the unified data with a selected format for further analysis. The key idea of this tool is to reproduce manual data consolidation and integration processes, and to help users to interact with extended $\mathrm{RaCoOn}$, providing a solution to integrate rail-related data and shorten the data purification and consolidation processes using ontologies. It aims to provide a projectindependent solution to help researchers and developers exploit the benefits brought by ontologies to the rail domain.

\section{CASE STUDY}

The work and findings described in Ortega et al. [8] and Armstrong et al. [9] are based primarily on two case study locations in Britain, the first on the East Coast Main Line 
between Newcastle and Edinburgh, and the second on the South West Main and Portsmouth Direct Lines between London Waterloo, Woking and Portsmouth Harbour stations. While the new, template- and ontology-based approach aims to replicate and generalise the processes used in Ortega et al. [8] and Armstrong et al. [9], there seemed little point in simply reproducing results already obtained (although checks for consistency will obviously be undertaken), and a new case study site was therefore identified and selected for the ongoing work. The chosen route is the "West Coastway" line along England's south coast between Portsmouth Harbour and Brighton stations, as shown in schematic form in Fig. 3.

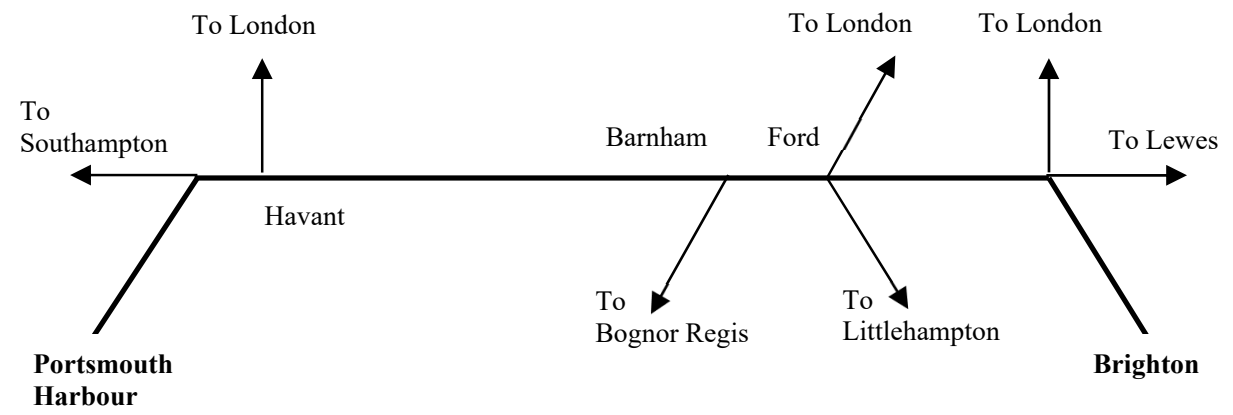

Figure 3: West Coastway schematic line diagram.

The length of the West Coastway is approximately 45.5 miles $(73.2 \mathrm{~km})$, and it is predominantly a two-track railway, with a few short, three-track sections. Line speeds along the route vary between $40 \mathrm{mph}$ and $85 \mathrm{mph}$ (approximately $64 \mathrm{~km} / \mathrm{h}$ to $137 \mathrm{~km} / \mathrm{h}$ ), with a $20 \mathrm{mph}$ (approximately $32 \mathrm{~km} / \mathrm{h}$ ) limit on the approaches to Brighton station. The route was chosen for a range of reasons, mainly because it is the subject of experimental and analytical activities in other research work being undertaken by members of the T2F team (see for example Young et al. [14]), and thus provides some potential synergies between activities.

\section{ONGOING AND FURTHER WORK}

Work is ongoing to develop and implement the ontology, and to replicate and extend the analytical processes described in Ortega et al. [8] and Armstrong et al. [9].

Looking beyond $\mathrm{T} 2 \mathrm{~F}$, the project team aspires to develop and implement a more generalised version of the modelling and analytical framework, ideally including other transport modes and the interdependencies both between modes and with other infrastructure systems, in a similar manner to the models developed by the ITRC [15]. Another possibility is to link the framework with DAFNI (Data and Analytics Facility for National Infrastructure) [16], allowing direct access to and integration with the growing range of infrastructure datasets and models stored on this facility.

\section{CONCLUSIONS}

As traffic levels on legacy railway networks increase, there is a growing need for interventions to maintain and renew the infrastructure (and ideally to increase the time intervals between interventions), and to reduce the environmental impacts of increasing traffic levels on users and, particularly, non-users of the railway. At the same time, the increasing traffic levels reduce the opportunities for access to the infrastructure, and so 
judicious selection from the available range of interventions is increasingly important if overall benefits are to be maximised.

Previous work in this area has demonstrated the feasibility and merits of applying CBA to individual interventions and to the comparison of alternative options, but the tools and methods used were comparatively situation-specific and inflexible. An improved, standardised and flexible assessment framework would therefore be very useful for enabling the rapid and consistent assessment of alternative interventions and/or interventions in different locations and operating circumstances, thus also facilitating comparisons between alternatives.

This paper describes the work undertaken to date in developing such a framework for future application, the case study chosen for its initial application and testing, the ongoing work in this area, and anticipated extensions to other projects and research domains in which the authors are engaged.

\section{ACKNOWLEDGEMENTS}

This research has been carried out by the authors as part of the EPSRC-funded TRACK21 (EP/H044949/1) and Track to the Future projects (EP/M025276/1). The authors are grateful to Network Rail for the provision of industry data, information and expertise.

\section{APPENDIX}

TRACK TO THE FUTURE INTEGRATED MODELLING INFORMATION TEMPLATE

Type of intervention:

Name of investigator:

Q1. Why will this intervention improve rail track systems?

Q2. In assessing the engineering impacts of this intervention what are the key input and output variables?

Q3. What are the main one-off (capital expenditure) and recurrent (operating expenditure) financial costs of the intervention?

What is your judgement of the magnitude of these costs and can they be quantified? If so, how? 
Q4. What are the main operator benefits? Operator refers to both infrastructure (Network Rail and its suppliers) and train services (TOCS and their suppliers).

What is your judgement of the magnitude of these benefits and can they be quantified? If so, how?

Q5. What are the main user benefits? User refers to customers of passenger and freight train services

What is your judgement of the magnitude of these benefits and can they be quantified? If so, how?

Q6. What are the main non-user benefits? Non-user refers to users of rival transport systems (air, road), residents or the wider community

What is your judgement of the magnitude of these benefits and can they be quantified? If so, how?

Any additional comments?

\section{REFERENCES}

[1] Bešinović, N. \& Goverde, R.M.P., Capacity assessment in railway networks. Handbook of Optimization in the Railway Industry, eds R. Borndörfer, T. Klug, L. Lamorgese, C. Mannino, M. Reuther \& T. Schlechte, Springer: Champaigne, pp. 2545, 2018.

[2] Cacchiani, V. \& Toth, P., Robust train timetabling. Handbook of Optimization in the Railway Industry, eds R. Borndörfer, T. Klug, L. Lamorgese, C. Mannino, M. Reuther \& T. Schlechte, Springer: Champaigne, pp. 93-115, 2018.

[3] ORR, Passenger train kilometres by operator: Table 12.13, 2020. https://dataportal.orr.gov.uk/media/1486/passenger-train-kilometres-by-operatortable-1213.xlsx. Accessed on: 19 Mar. 2020.

[4] ORR, Freight train kilometres by operator: Table 13.25, 2020. https://dataportal.orr.gov.uk/media/1459/freight-train-kilometres-by-operator-table1325.xlsx. Accessed on: 19 Mar. 2020.

[5] Armstrong, J. \& Preston, J., Balancing railway network availability and engineering access. Proceedings of the Institution of Civil Engineers - Transport, 2019.

DOI: $10.1680 /$ jtran.19.00045. 
[6] Track21, Railway track for the 21st century. http://track21.org.uk/. Accessed on: 25 Feb. 2020.

[7] Track to the Future, https://t2f.org.uk/. Accessed on: 25 Feb. 2020.

[8] Ortega, A., Blainey, S., Preston, J., Thompson, D., Squicciarini, G., Ntotsios, E. \& Armstrong, J., Noise reduction for ballasted track: a socio-economic assessment. WIT Transactions on Ecology and the Environment, vol. 181, WIT Press: Southampton and Boston, pp. 461-472, 2018. DOI: 10.2495/CR180411.

[9] Armstrong, J., Ortega, A., Blainey, S., Preston, J., Thompson, D., Squicciarini, G. \& Ntotsios, E., Noise reduction for ballasted track: A comparative socio-economic assessment. International Journal of Transport Development and Integration, 3(1), pp. 15-29, 2019. DOI: 10.2495/TDI-V3-N1-15-29.

[10] HM Treasury, The Magenta Book. www.gov.uk/government/publications/themagenta-book. Accessed on: 25 Feb. 2020.

[11] Morris, C. \& Easton, J., Use of ontology for data integration in a degraded mode signalling system. WIT Transactions on Ecology and the Environment, vol. 181, WIT Press: Southampton and Boston, pp. 215-223, 2018. DOI: 10.2495/CR180191.

[12] Department for Transport, WebTAG, 2020. www.gov.uk/guidance/transport-analysisguidance-webtag. Accessed on: 18 Feb. 2020.

[13] Tutcher, J., Easton, J. \& Roberts, C., Enabling data integration in the rail industry using RDF and OWL: The RaCoOn ontology. ASCE-ASME Journal of Risk and Uncertainty in Engineering Systems, Part A: Civil Engineering, 3(2), pp. F4015001-1-F401500112, 2017. DOI: 10.1061/AJRUA6.0000859.

[14] Young, M., Rempelos, G., Ntotsios, E., Blainey, S., Thompson, D. \& Preston, J., A transferable method for estimating the economic impacts of track interventions: application to ground-borne noise reduction measures for whole sections of route. Journal of Rail and Rapid Transit (submitted).

[15] Lovric, M., Blainey, S. \& Preston, J., A conceptual design for a national transport model with cross-sectoral interdependencies. Transportation Research Procedia, 27, pp. 720-727, 2018.

[16] DAFNI, 2020. www.dafni.ac.uk/. Accessed on: 19 Feb. 2020. 